\title{
Minimal invasive and effective therapy of faecal incontinence with a preanal tape
}

\author{
(1) SIDI MUCTAR ${ }^{1}$, (1) ANDREI I MÜLLER-FUNOGEA², (1) MARTIN KOWALLIK³ , (1) NICOLAS FISCHER ${ }^{4}$ \\ 1Department of Urogynaecology, Helios Kliniken Krefeld, Lutherplatz, Germany \\ 2Department of Gynaecology, St-Antonius Hospital Eschweiler, Germany \\ ${ }^{3}$ Department of Coloproctology, Magen-Darm-Zentrum Wiener Platz, Cologne, Gemany \\ ${ }^{4}$ Department of Urology, Universitätskliniken Köln, Cologne, Germany
}

\begin{abstract}
Objective: The existing surgical therapy modalities for the treatment of faecal incontinence do not provide satisfactory results. Our understanding of the pelvic floor anatomy and physiological biomechanics of the anal canal are the basis for the theoretical development and practical therapy of faecal incontinence.

Materials and Methods: According to the Integral Theory System and similarly to a suburethral sling, a preanal sling is placed under the pelvic floor.

Results: The presented operation was successfully done on three patients beyond treatment for their faecal incontinence.

Conclusion: We present a simple and functional surgical method for the treatment of faecal incontinence.

Keywords: Anatomy; faecal incontinence; sphincter; minimal invasive surgery; rectum
\end{abstract}

\section{INTRODUCTION}

Faecal incontinence is defined by involuntary, uncontrolled faecal loss. The prevalence of faecal incontinence is high. In the literature the prevalence is: $7-15 \%$ in the general population, in hospitals up to $30 \%$ and in nursing homes up to $70 \%{ }^{1}$ The number of unreported cases is probably higher, as patients do not report it spontaneously and most doctors do not specifically ask about faecal incontinence routinely during the medical history survey. The causes that lead to faecal incontinence are manifold. Local anatomical changes affecting the sphincter ani, pelvic floor muscles, ligaments, fascia and their visceral and somatic innervations can be causes of faecal incontinence. Diseases of the peripheral and central nervous system can also cause defecation problems and faecal incontinence. Accurate anamnesis, examinations such as perineal sonography, anorectal manometry, rectoscopy, digital rectal examination, as well as the dynamic pelvic floor magnetic resonance imaging (MRI), are important assessment tools for the anatomy and function of the anal canal. ${ }^{2}$

Therapy modalities vary from conservative to surgical. Bharucha et al. ${ }^{3}$ list for the therapy of faecal incontinence "15 best practice advice", of which 13 practices are classified as surgical interventions. ${ }^{3}$

This list shows that there is no best surgical solution for the treatment of faecal incontinence. It is therefore of uttermost importance to develop a reliable therapy for faecal incontinence. ${ }^{4}$ 
We present a simple and functional surgical method for the treatment of faecal incontinence.

\section{MATERIALS AND METHODS}

Three patients suffering from faecal incontinence were submitted to this new operation. All three underwent multiple conservative therapies and operations without satisfactory results. A colostomy was finally indicated for all these patients as the lesser evil choice. We offered our new operation as an alternative possibility for them to avoid colostomy.

We explained the anatomical and physiological principles of this operation, the operation itself and the preliminary experiments on corpses performed anteriorly. The operation is performed in dorsal lithotomy position with intraoperative antibiotic administration as a single-shot with metronidazole and ceftriaxone. The principles of this operation are based on the integral biomechanical movements of the anal sphincter and the pelvic floor muscles. The operation itself is performed below the pelvic floor on the sphincter ani while integrating the ligaments.

\section{Anatomy}

Undisturbed and intentionally controlled flow and retention of stool through the anus are ensured by intact pubococcygeus (PCM), puborectalis (PRM), longitudinal anal (LAM) and sphincter ani muscles. For support, the sacrouterine ligaments (SUL), the rectovaginal fascias and the perineal body according to the musculo-elastic theory of Prof. Peter Petros play an important role in the function of the anorectal canal. ${ }^{5}$ The PCM stretches from the bottom edge of the os pubis to the os coccygeus. It is divided in two components. The front component is responsible for opening and closing the bladder during micturition. The posterior component, the levator plate (LP), is responsible for opening the canal.

At the point where the two components connect, two circular openings are formed. The front opening is the hiatus genitalis, the posterior one is the hiatus analis. In the hiatus analis, the rectum runs downwards and merges with the sphincter ani. At this point, the rectum is connected anteriorly to muscle cords of the PCM. These run downwards and form the frontal LAM. ${ }^{6}$ Posteriorly, the rectum is connected to branches of the LP. These branches form the posterior part of the LAM (Figure 1).

Below the PCM lies the PRM. It comes from the lower edge of the symphysis, circles around the posterior surface of the rectoanal compound and runs back in the opposite direction to the symphysis, where it is attached.

A very important muscle for the function of the canal, which is sometimes ignored, is the LAM. This muscle runs down through the hiatus analis, inter-sphincterically to the anodermal connective tissue, where it is attached. Parts of his muscle fibres emerge from the sphincter ani externus wall and run with the preand post- anal ligaments. They anchor anteriorly in the perineal body and posteriorly with the os coccygeus. The main function of the LAM is to widen the canal during defecation, while at the same time, the PRM relaxes and the PCM simultaneously clamps with the LP in the opposite direction. The sphincter ani and the pelvic floor muscles are innervated with somatic nerve branches of the N. pudendus. The rectum wall and its longitudinal and circular muscles are innervated with parasympathetic nerves (Figure 2).

\section{Physiology}

In the literature, the causes of stool incontinence are manifold and reported as multifactorial. Based on the musculo-elastic theory, we present here a contribution to clarify the dynamics of the defecation process. The anal canal complex consists of the external and internal sphincters, the LAM, connective tissue, nerves, vessels and mucosa. The LAM runs topographically between external and internal sphincter. They function as a unit. They are innerved through somatic and visceral nerve branches. The anal canal complex as a defecation and retention unit includes the pelvic floor muscles, ligaments and fascia, which

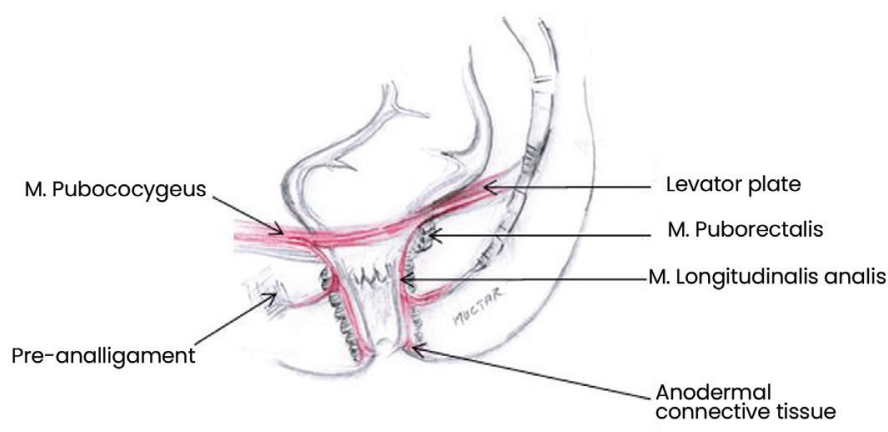

Figure 1. Course of the LAM between the internal and external sphincter, through the pre- and postanal ligaments and anchoring to the anodermal connective tissue

LAM: Longitudinal anal

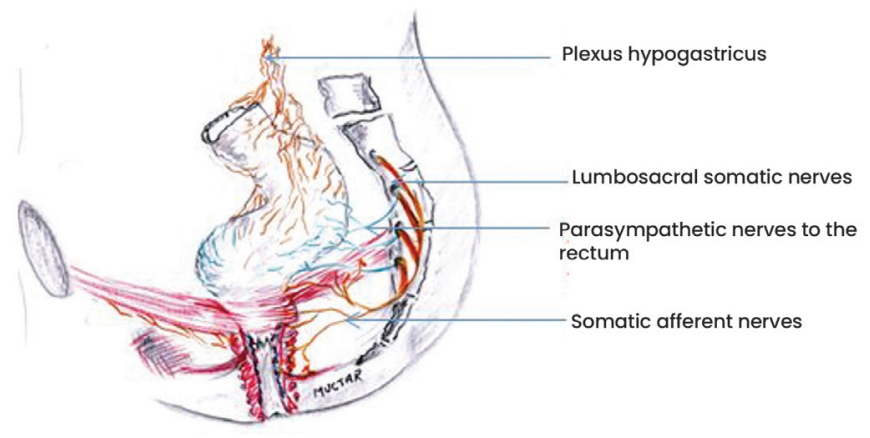

Figure 2. Description of the innervation of the pelvic floor 
interact synchronously and temporarily with the sphincter ani. This unit can be disturbed by changes in position, lesions and degeneration of the individual components. From the results of the literature on diagnostics, such as dynamic MRI defaecography, rectal manometry, pudendal and tibial nerve conduction studies, the course of defecation is interpreted here as follows:

At rest, the PCM and the LAM are relaxed. The PCM, the internal sphincter ani and in combination with a relaxed ampoule recti, they are minimally stretched, so that no air or stool can be lost incidentally. ${ }^{7}$

During defecation, the PCM is contracted bidirectionally. The PCM has two components. The anterior part of the PCM moves forward towards os pubis (Figure 3). The LP moves posteriorly towards the os sacrum. Thus, the LAM, which originates from these muscles and runs caudally between the internal and external anal sphincter is stretched. This stretching expands the anal canal circumference, hence opening the canal.

This opening is supported by the relaxation of the PRM and the external sphincter ani. This is followed by the expulsion of the faeces from the ampoule recti, which contracts during defecation. A contraction of the puborectal muscle pulls the cranial part of the anal canal forward, while the lower part of the anal canal, fixed by the pre- and post-anal ligaments, is only slightly displaced cranially. The position and function of the anal canal are ensured by ligaments and connective tissue [pre- and post- anal ligaments, SUL/rectovaginal fascias (RVF)], which are drawn in blue (Figure 3). If ligaments and connective tissue are weakened or damaged, the muscles cannot fully perform their

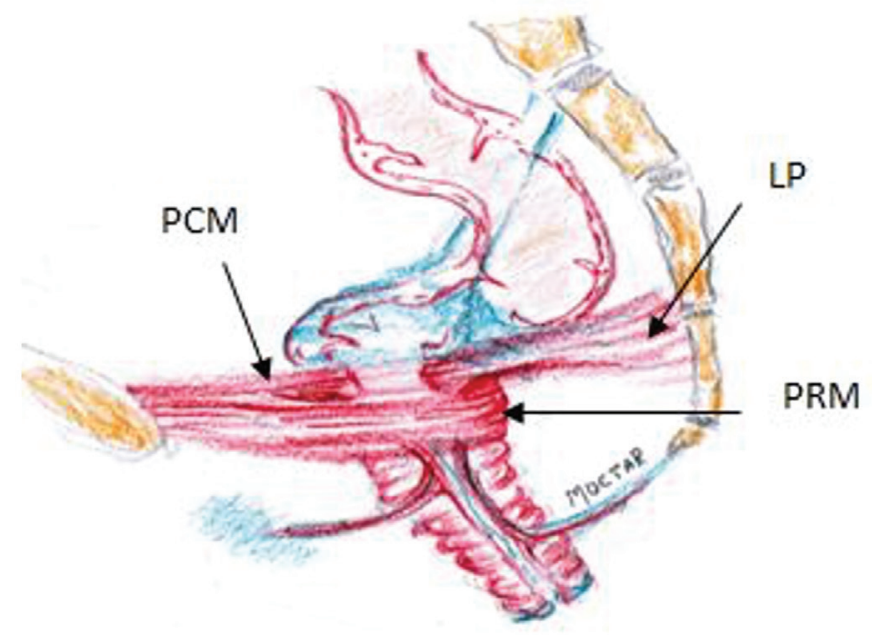

Figure 3. Shows the inclination of the anal canal towards the front in a situation of a filled ampoule recti

PCM: Pubococcygeus muscle, LP: Levator plate, PRM: Puborectalis muscle assigned functions. The consequences are prolapse of the pelvic organs up to stool and/or urine loss. ${ }^{8}$ When the puborectal muscle is taut, it pulls the anal canal forward by $20^{\circ}$. This forward inclination, which can also be seen dynamically during exertion or cough in perineal sonography, led us to believe that stabilizing the anal canal against the traction of the puborectal muscle could lead to a sufficient change in pressure in the lumen of the anal canal. This insight led to the preanal surgery method we describe and has proven to be effective for treating stool incontinence due to insufficient sphincter pressure.

Own observations are confirmed in various studies according to which lesions or weakening of LAM alone or in combination with the SUL can cause dysfunctions of the rectoanal canal such as constipation or incontinence. ${ }^{9}$ Therefore, in stool incontinence with clinically detectable prolapse genitalis or in rectoceles the correction of ruptures or relaxations of the SUL should also be considered.

Figure 4 demonstrates the physiology and pathophysiology of stool continence. The yellow mark shows where all muscle forces meet for the physiological closure of the rectal canal. If the bowel movement is interrupted, the ileococcygeus muscle contracts towards the os coccygeus. Together with the LP, it pulls the rectovaginal fascia diagonally backwards so that the rectoanal angle is stressed. The PRM is contracted and narrows the anal canal. SUL, RVF and PB form a unit to harmonize the function of the pelvic floor muscles.

Seen from above, the PCM is the first muscle layer of the pelvic floor muscles. It consists of two components. The anterior part lies between the os symphysis and the rectum, the posterior component, also known as the LP, lies between the rectum and the os coccygeus. When holding and during defecation, both components can move in the same direction as required or independently pull in opposite directions. This explains why we can only initiate defecation or micturition as well as both at the same time.

As soon as there is an urge to defecate, the PFM and anal sphincter muscles will act according to their function. Under normal physiological conditions, the stool can be held or emptied at will.

Helpful diagnostic tools include anorectal tonometry, perinealand endoluminal anal sonography for assessing the anatomy and function of the sphincter. The dynamic pelvic floor MRI supports the assessment of both the anatomical structural intricacies as well as the functional anatomy of the pelvic floor and the pelvic organs.

There are different methods to graduate the severity of faecal incontinence. However, in the hands of the experienced 


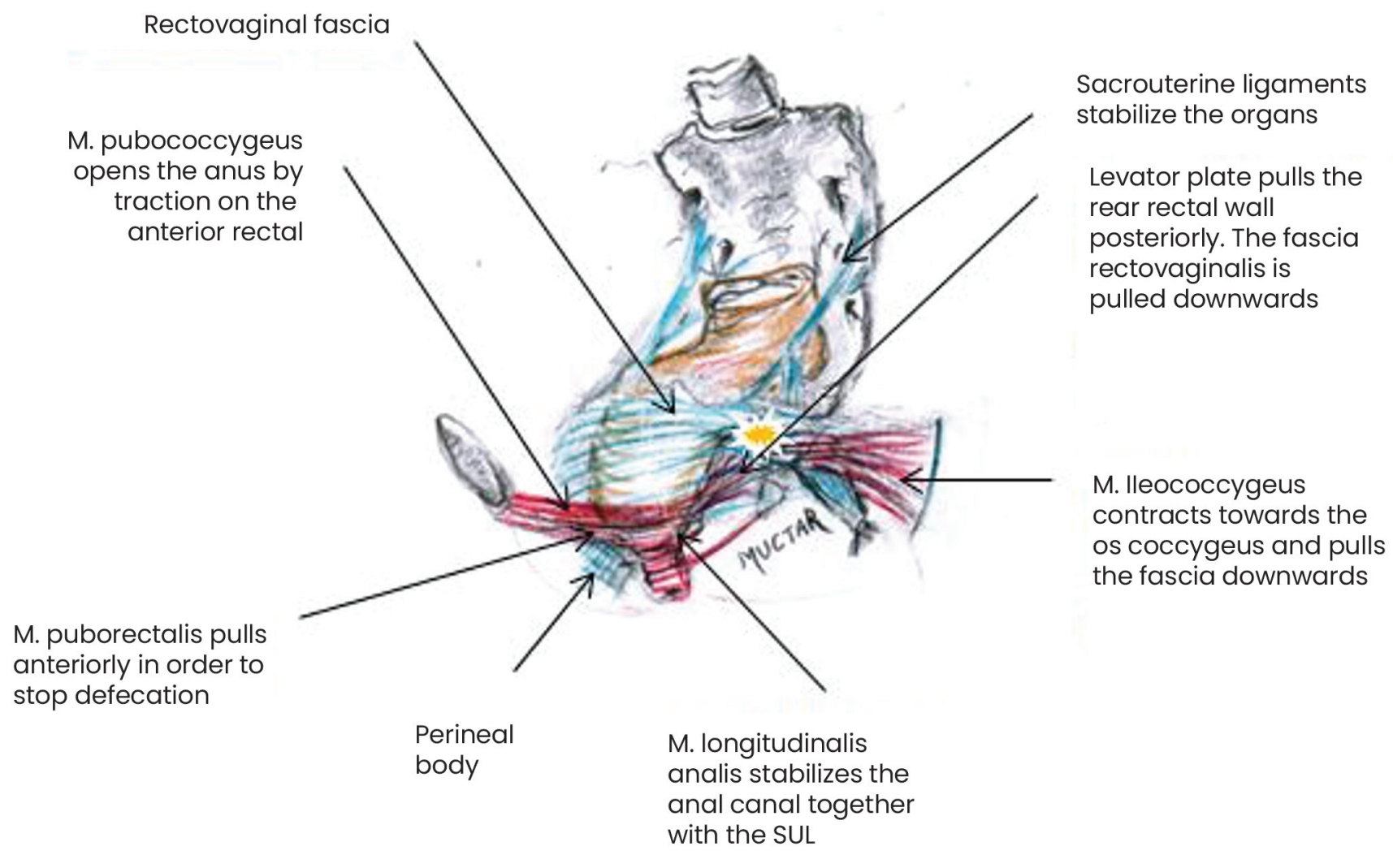

Figure 4. Shows the physiology and pathophysiology of stool continence. The yellow mark shows where all muscle forces meet for the physiological closure of the rectal canal

SUL: Sacrouterine ligaments

investigator, digital rectal examination is a safe and sufficient method for estimating the tonus of the sphincter ani muscle. ${ }^{10}$ This, in combination with a comprehensive history, is an important indicator in decision-making for further examinations as well as for the treatment of sphincter ani incontinence.

The results of surgical treatment for deep-seated rectal cancer have recently improved considerably due to advances in technology. However, the consequences of the operation have not been optimally resolved. Obviously, deep rectal resection carries the risk of postoperative stool incontinence. ${ }^{11}$ The illustration (Figure 5) shows the fine anatomy of the anal canal. Accordingly, the narrow space along the anal canal between the LAM and the LRM (longitudinal rectal muscle) should be accurately dissected during the operation. In our understanding of the dynamics and physiopathology of the recto-anal complex, this space is the path of dissection for deep rectal resection to ensure continence. The LAM is an arbitrarily controlled component. The LRM is a component controlled by the parasympathetic nervous system. Both muscles are very important units for anal continence. With deep rectal resection one should consider the relationship between muscles and avoid parasympathetic nerve branches.

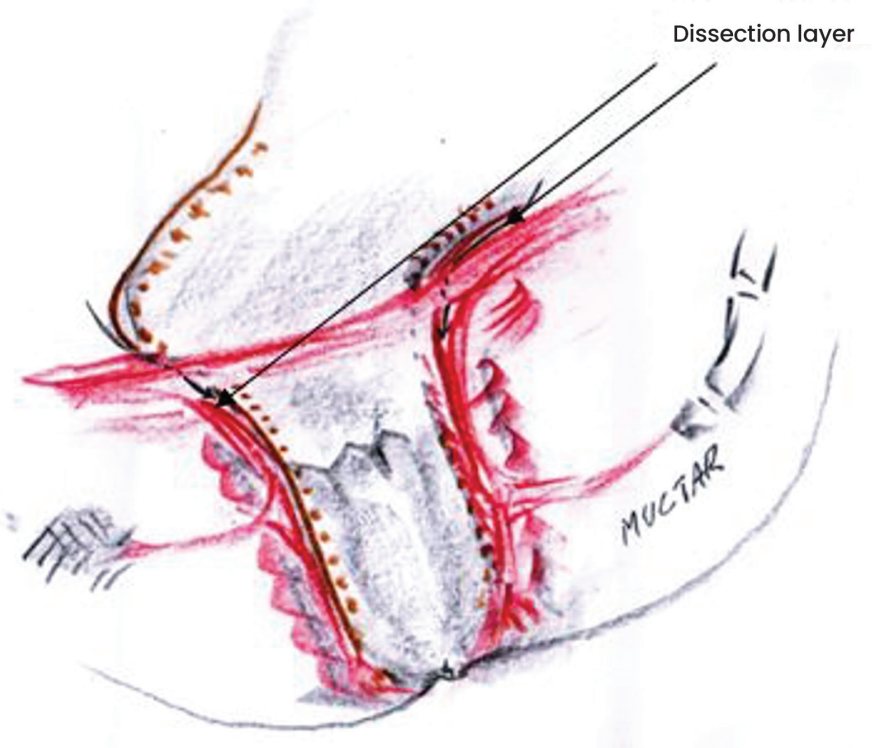

Figure 5. The illustration shows the fine anatomy of the anal canal and the dissection layer of the operation. The illustration shows the fine anatomy of the anal canal. The narrow space along the anal canal between the LAM and the LRM should be accurately spared during resections for low rectal tumors

LAM: Longitudinal anal muscle, LRM: Longitudinal rectal muscle 


\section{Surgical technique}

Before performing this innovative surgical technique in vivo, we first performed an experimental operation on a female corpse at an urogynaecological workshop. In the following the U-shaped pre-anal tape system as a sphincter support was carried out in three highly faecal incontinent patients. The operation takes place below the pelvic floor. Therefore, the organs that lie above the pelvic floor are not endangered by the operation. An additional advantage of this technique is in addition to the fixation of the canal, that a stabilization of the pelvic floor can be achieved.

\section{Operation description}

The operation is carried out in dorsal lithotomy position with intraoperative antibiotic intravenous administration as a singleshot of Metronidazole and Ceftriaxone. The index is inserted into the anal canal to assess the resting pressure of the canal. The finger then pushes the middle portion of the canal forward so that the correct cutting site on the perineum can be estimated (Figure 6).

A paraanal cavum is then dissected bilaterally below the pelvic floor with scissors. A tunnel to the spina ischiadica is then formed using digital blunt dissection, (Figure 7). The tape is attached three centimetres medially from the spina on the sacrospinal ligaments (Figure 8 and 9).

There are various techniques for fixing the tape at this point, depending on the system used. We use either an I-Stitch or the $\mathrm{TFS}^{\circ}$ system. The $\mathrm{TFS}^{\circ}$ system has the advantage of being still adjustable after anchoring it to the sacrospinal ligaments, so

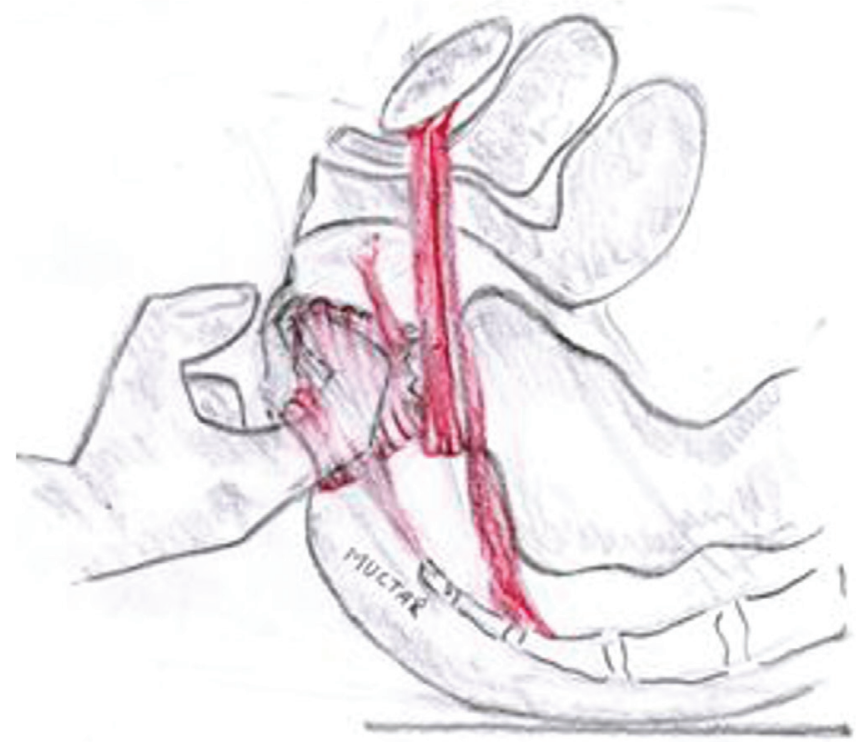

Figure 6. Assessing the location of the incision on the perineum with the thumb that the tension can be set to the desired pressure. The required pressure can be estimated digitally or, better using manometry at $40-60 \mathrm{CmH}_{2} \mathrm{O}$ (Figure 10). Alternatively, the tape can be applied through a pararectal access as in prolapse- and rectocele correction. Here, the procedure takes place below the pelvic floor as well.

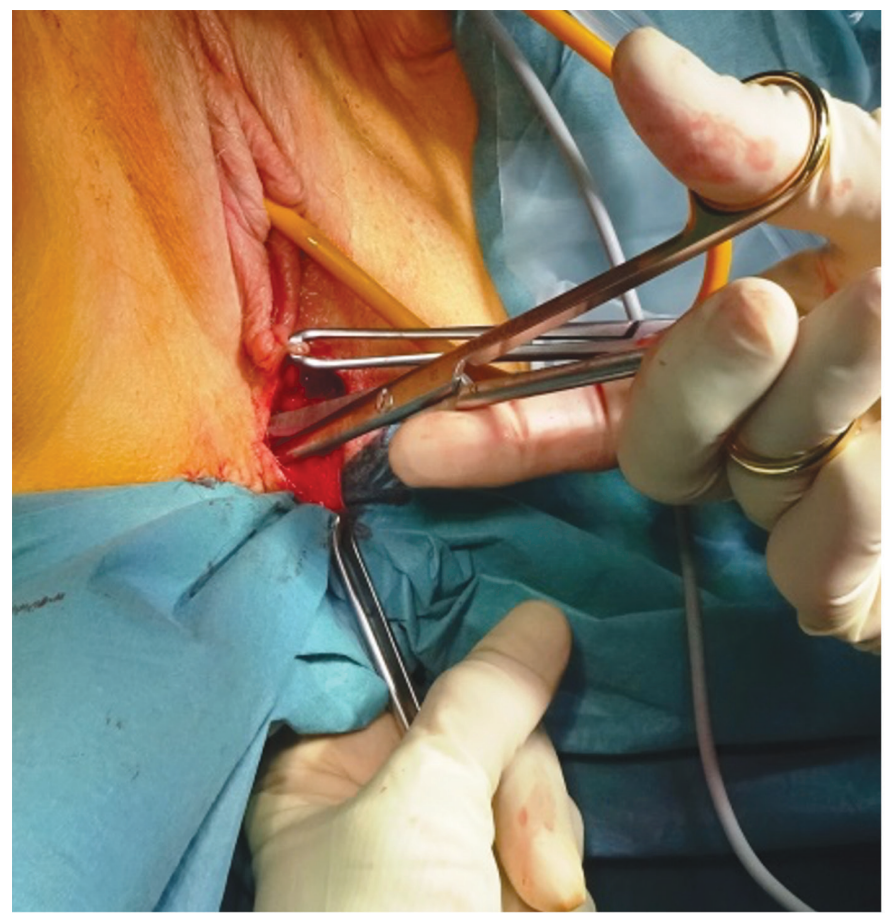

Figure 7. Dissection of the paraanal space with scissors

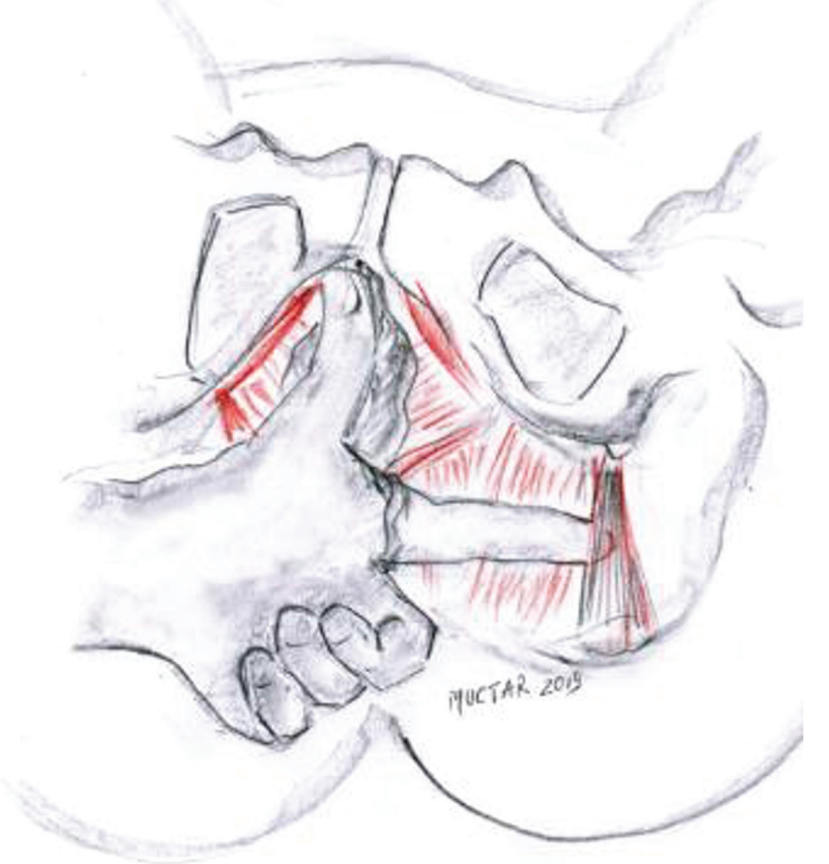

Figure 8. Palpation of the sacrospinal ligament with a finger and choosing a suitable position for the tape. Cave: The finger runs below the Pelvic floor muscles 
For the execution of this surgical technique we used medically approved non-coated polypropylene tapes, such as the suburethral tape [trans vaginal tape (TVT) or transobturator tape (TOT)] in urine incontinence. No additional approval of the ethics committee was therefore required.

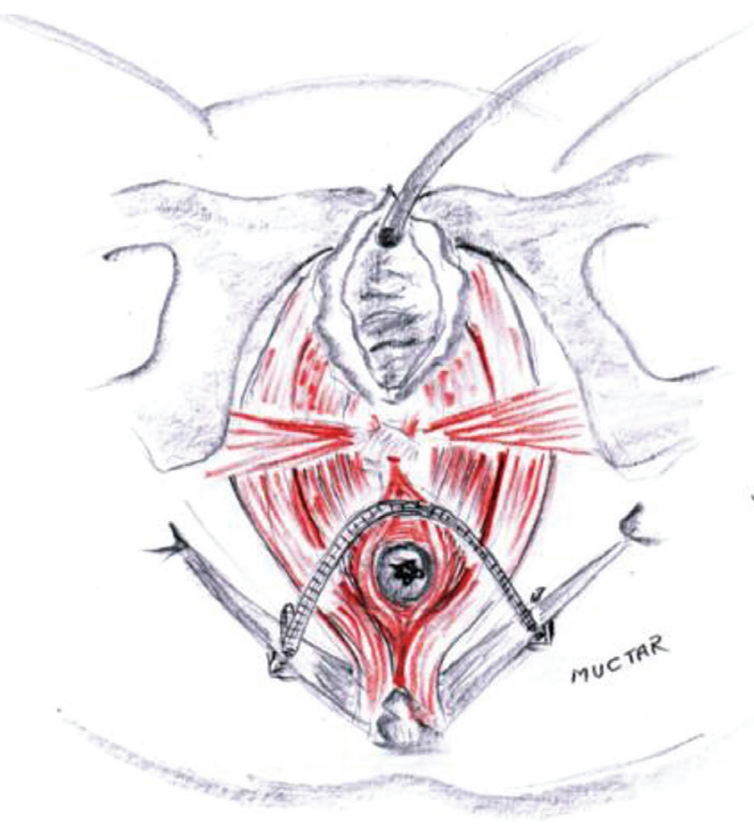

Figure 9. Final position of the tape on the front circumference of the anal canal. The wound is then closed

\section{Patients}

Three patients underwent this operation. All three of them suffered from faecal incontinence and were ultimately to be submitted to a colostomy. Prior to our operation, they experienced unsuccessful conservative, dietary and surgical measures and were declaredly beyond treatment. Before colostomy as a final procedure, we offered the operation aiming at a support of the anal canal with a U-shaped sling. All patients approved after extensive informed consent.

\section{RESULTS}

The sphincter tonus to graduate the severity of faecal incontinence was estimated by the digital rectal exam and in combination with a comprehensive medical history, was an important indicator in the decision-making process for the treatment of sphincter ani incontinence.

Through intensive study of the dynamic pelvic floor MRI, we were able to observe how the pelvic floor muscles interact with the sphincter ani. From these observations, we developed a theory of a functionally oriented surgical method, and the operation on a corpse was found to be simple and feasible. In addition to a second-degree recurrent rectocele with a weak perineal body, one patient (patient 2) also had a general connective tissue weakness, known as Marfan syndrome. Intraoperatively,

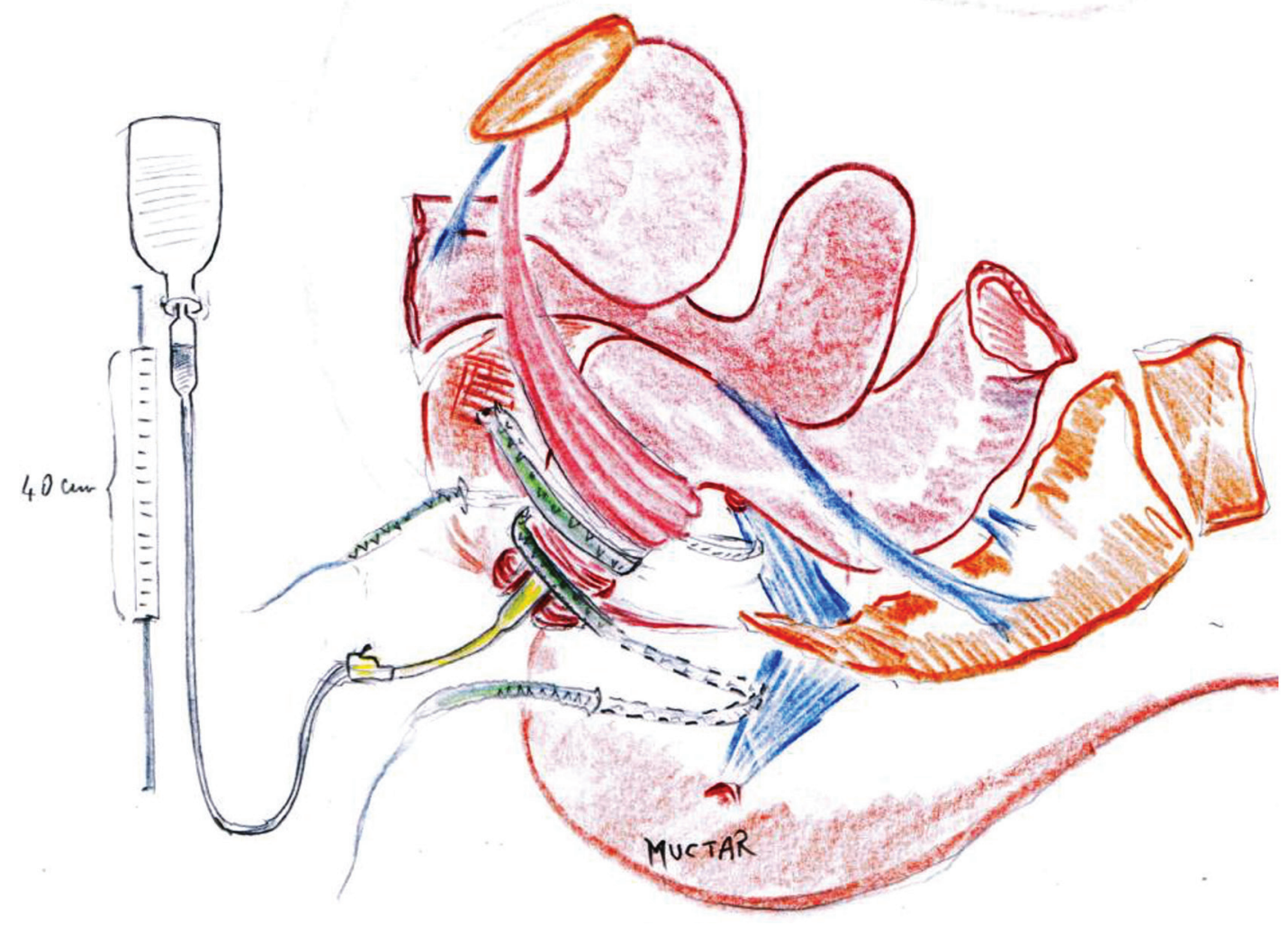

Figure 10. A transanal manometer is used to assess the intra-anal pressure. Alternatively, this can also be done by placing a finger in the anal canal. This latter method depends on the experience of the surgeon 
we found in this patient that her pelvic connective tissue and sacrospinal ligaments were not sufficiently resistant to securely attach the anchor. In this case, we performed a modified version of the U-tape attaching it additionally on the posterior anal circumference. The intraanal pressure measurement showed sufficient pressure for holding stool. Postoperatively, this patient developed complete stool retention. The following day, we were able to detect an almost normal pressure of the anus during the rectal digital examination. However, during the defecation attempt, we noticed a narrowing of the canal, which dissolved at rest. Therefore, we had to readjust the tension of the tape. The patient was discharged the following day without complications. The postoperative follow-up after four months showed no negative effect of the tape on the canal. The patient is still continent to date.

Both two other patients did not show any complications postoperatively. Four months after demission, they were both continent and satisfied with the results. During postoperative inspection and digital examination, we could not detect any anatomical or pathological changes in the canal.

\section{DISCUSSION}

Stool continence is ensured by synchronous interaction of muscles, ligaments and fascias. These are the PRM, the PCM, the LAM, the SUL, the perineal body and the sphincter ani (Figure 3). All these components are innervated by somatic nerve branches of the N. pudendus and vegetative nerve branches on both sides (Figure 4). Other components such as the compliance of the rectum volume and the mental perception of the stool process have a major influence on the regulation of the bowel motion.

The position and relationships of the pelvic organs to each other can be recognized and assessed excellently by perineal sonography in dorsal lithotomy position. The dynamics and the directions of movement of the anorectal section are easily interpretable.

The canal is tilted forward at rest around $20^{\circ}$. It is secured in this position by the pre- and postanal musculoelastic ligaments as well as the LAM muscles. In the relaxed state, the pelvic floor muscles and the pre- and postanal ligaments secure the position and inclination of the canal forwards. Under tension, the PRM muscle loop pulls together with the preanal ligament on the ano-rectal angle, which narrows the rectum and pulls the anus forwards. The preanal ligament is firmly connected with the perineal body. Loosening of the ligament is usually caused by lesions of the perineal body, such as trauma at birth. The loosening of the preanal ligaments in combination with other components functionally important for undisturbed defecation can lead to destabilization of the topography of the canal and subsequently to stool incontinence. These observations, supported by perineal sonography and dynamic pelvic floor MRI, led us to a theory aiming at the stabilization of the canal. Therefore, in non-sphincterian stool incontinence with clinically detectable prolapse genitalis or in rectoceles the correction of ruptures or relaxations of the SUL should also be considered. The literature states that common surgical methods for the treatment of this type of stool incontinence such as fillers, muscle interponates, artificial sphincters or sacral neuro stimulation do not show statistically good and reliable results. ${ }^{12}$ We have found both in corpse preparation and in vivo, that the prototype we have designed in extension to the Integral Theory System to stabilize the pre-anal ligament leads to a stabilization and increase of pressure.

According to the integral theory of Prof. Peter Petros and Prof. Ulmsten, stress urinary incontinence could be treated through stabilization of the urethra by strengthening the pubourethral ligaments. The TVT as a minimally invasive procedure was introduced 30 years ago. ${ }^{13}$ Similarly, the preanal tape works as a minimally invasive therapy for stool incontinence. The fixation and repositioning of the canal by means of a tape below the pelvic floor muscles leads to reliable stool continence. ${ }^{14}$

The aim of the operation is to stabilize the anal canal with a broadly open U-shaped pre-anal tape fixed to the sacrospinal ligaments. Access is provided by a perineal section. The fixation of the preanal ligament by this technique also leads to the lifting and stabilization of an existing pelvic floor muscle relaxation. The tape position is easily verifiable so that it may be simply removed in case of possible early or late complications.

\section{CONCLUSIONS}

The preanal tape is a simple, fast and effective method in the treatment of faecal incontinence especially with atone sphincter ani because it drives correctly the closing forces of the pelvic floor muscles to the rectum. Without providing long-term results, we hope to have provided a small contribution to the existing treatment options. Studies to develop a suitable proprietary device for the aims of this technique were already started by us.

\section{Ethics}

Informed Consent: All patients approved after extensive informed consent.

Ethics Committee Approval: No additional approval of the ethics committee was therefore required.

Peer-review: Externally peer-reviewed. 


\section{DISCLOSURES}

Conflict of Interest: The authors have no conflicts of interest.

Financial Disclosure: The authors declared that this study has received no financial support.

\section{REFERENCES}

1. Frieling T. Incontinence - Etiology, diagnostics and Therapy. Dtsch Med Wochenschr 2016; 141: 1251-60.

2. Prichard DO, Lee T, Parthasarathy G, Fletcher JG, Zinsmeister AR, Bharucha AE. High-resolution Anorectal Manometry for Identifying Defecatory Disorders and Rectal Structural Abnormalities in Women. Clin Gastroenterol Hepatol 2017; 15: 412-20.

3. Bharucha AE, Rao SSC, Shin AS. Surgical Interventions and the Use of Device-Aided Therapy for the Treatment of Faecal Incontinence and Defecatory Disorders. Clin Gastroenterol Hepatol 2017; 15: 1844-54.

4. Saldana Ruiz N, Kaiser AM. Faecal incontinence - Challenges and solutions. World J Gastroenterol 2017; 23: 11-24.

5. Petros PP, Swash M. A Musculo-Elastic Theory of anorectal function and dysfunction in the female. Pelviperineology 2008; 27: 86-7.

6. Muro S, Yamaguchi K, Nakajima Y, et al. Dynamic intersection of the longitudinal muscle and external anal sphincter in the layered structure of the anal canal posterior wall. Surg Radiol Anat 2014; 36 : $551-9$.

7. Bharucha AE, Fletcher JG, Harper CM, et al. Relationship between symptoms and disordered continence mechanisms in women with idiopathic faecal incontinence. Gut 2005; 54: 546-55.

8. Petros PE. Cure of urinary and faecal incontinence by pelvic ligament reconstruction suggests a connective tissue etiology for both. Int J Urogynecol 1999; 10: 356-60.

9. Muctar S, Schmidt WU, Batzill W, Westphal J. Functional anatomy of the female pelvic floor: interdisciplinary continence and pelvic floor surgery. Urologe A 2011; 50:785-91.

10. Tantiphlachiva K, Rao P, Attaluri A, Rao SS. Digital rectal examination is a useful tool for identifying patients with dyssynergia. Clin Gastroenterol Hepatol 2010; 8: 955-60.

11. Rubinkiewicz M, Zarzycki P, Witowski J, et al. Functional outcomes after resections for low rectal tumors: comparison of Transanal with laparoscopic Total Mesorectal excision. BMC Surg 2019; 19: 79.

12. Brunner M, Bittorf B, Matzel K. Modern Strategies for the Treatment of Faecal Incontinence. Zentralbl Chir 2019; 144:190-201.

13. Petros PE, Ulmsten U. An Integral Theory of female urinary incontinence. Acta Obstet Gynecol Scand 1990; 153: 1-78.

14. Muctar S. Atlas der urogynäkologischen und proktologischen Operationstechniken. 1st edition. Georg Thieme Verlag, 2018.

\section{Multidisciplinary UroGyneProcto Editorial Comment}

To improve the integration among the three segments of the pelvic floor, some of the articles published in Pelviperineology are commented on by Urologists, Gynecologists, Proctologists/Colo Rectal Surgeons or other Specialists, with their critical opinion and a teaching purpose. Differences, similarities and possible relationships between the data presented and what is known in the three fields of competence are stressed, or the absence of any analogy is indicated. The discussion is not a peer review, it concerns concepts, ideas, theories, not the methodology of the presentation.

Uro... Gyneco... Procto... Other...

Uro... The correction of fecal incontinence by applying a preanal tape represents an interesting extension of the integral theory of Peter Papa Petros to the posterior compartment of the pelvic floor. The mini-invasiveness of the procedure is quite appealing, though the understanding of the principle according to which this solution should be effective is not immediate. It seems that this technique may produce the same initial sensations of amazement experienced by urologists in the 90s with the proposal of applying a suburethral tape in women with stress urinary incontinence, a technique that was then quickly acquired worldwide. For various causes, stress urinary incontinence derives essentially from the laxity of the vagina or its supporting ligaments due to an intrinsic alteration of collagen. In this context, the pelvic floor muscles do not perform the function of producing direct forces with a stretching of the pelvic organs, as usually happens thanks to the pubococcygeus muscle (PCM), the levator ani and the longitudinal striated muscle of the anus, resulting in failure to stabilize the sphincter activity of the urethra. Similarly, the anal canal complex, as a defecation and retention unit, includes pelvic floor muscles, ligaments and fascia which interact synchronously with the anal sphincter. In this way, the fixation of a tape to the sacrospinal ligaments restores the harmonious functioning of the PCM and internal sphincter of the anus which in resting conditions are minimally stretched in such a way as to prevent the involuntary loss of stool. The intuition of 
the authors in applying some principles of the pathophysiology of urinary continence to the posterior pelvic floor is absolutely fascinating, though it requires clinical confirmation over time and with a more significant number of cases.

SALVATORE SIRACUSANO

Professor of Urology, University of L'Aquila, Italy salvatore.siracusano@univr.it

Procto... Anal continence is an extremely complex function based on many factors, some still not fully understood. Unlike urinary continence, the anus must be able to contain and expel a solid, liquid and gaseous content at a desired time and place, a much more demanding task than that of the urinary excretory system. Muctar and Coll.'s research fills with enthusiasm and hope those who trust the Integral Theory (IT) that has allowed both the understanding of the importance of the ligaments of the female pelvis in the onset of urinary incontinence, and to correct it in an apparently very simple way. "In pelvic floor dysfunctions it is typical that anorectal, bladder and pain symptoms often are coexistent. This is because the same ligaments and muscles within the pelvis are responsible for the different functions while ligamentous laxities or shrinkage are responsible for dysfunctions which often are co-existing" (Bernhard Liedl, personal communication). For the treatment of faecal incontinence we have experienced the frustration of experimenting with many surgical techniques, even very expensive ones such as the electrostimulated graciloplasty or the artificial sphincter, with very poor results. It was also tried to apply a sling to support the puborectalis muscle following its course (Latorre F, ....Cervigni M. Pelviperineology 2013; 32: 9-13), without any significant long term success having not correctly interpreted the principles of IT. The conclusion was drawn that, apart from the reconstruction of the sphincters interrupted by a trauma of various types, the solutions to try to improve the quality of life of patients with fecal incontinence consist mainly in rehabilitation or in Trans Anal Irrigation (TAI). The aim of the operation described in this article is to stabilize the anal canal with a broadly open U-shaped pre-anal tape fixed to the sacrospinal ligaments, so the two backward vectors can better rotate the rectum around the $U$ sling to close it. This work now opens up an extremely interesting path, albeit with the limitation of having only been tested in a small number of female patients. Among the many important elements of the research of Muctar and Coll., in addition to the cleverness of the technique, should be noted the multidisciplinary nature of the Authors who have tried this approach, colorectal surgeons and urogynecologists: a truly interdisciplinary technique. And, once again, Liedl considers that "we are now moving from being guided by Integral Theory to a true Integral Practice increasingly confirmed by evidence-based knowledge. After all, in the footsteps of Karl Popper, the function of a good theory is to offer us a model that must always be corroborated by experimental evidence." We have created more and more evidence for cure of women from their co-existing symptoms by appropriate surgery. So rather than on a theory we should concentrate on the scientific basis of functional anatomy, pathophysiology, surgical principles, which all should be integral".

GIUSEPPE DODI Colorectal Surgeon, University of Padova, Italy giuseppe.dodi@unipd.it 